Brit. F. industr. Med., 1966, 23, 210

\title{
Peripheral Neuropathy in Rats Produced by Acrylamide
}

\author{
PAMELA M. FULLERTON and J. M. BARNES \\ From the Toxicology Research Unit, M.R.C. Laboratories, Carshalton, Surrey, \\ and the Institute of Neurology, Queen Square, London
}

Acrylamide produces ataxia and limb weakness in rats when given in repeated oral doses of 25 to $100 \mathrm{mg}$. $/ \mathrm{kg}$. at various intervals or mixed with the diet in concentrations of 100 to 400 p.p.m.

An electrophysiological and histological study of peripheral nerves has been carried out in chronically poisoned rats. In animals with severe clinical abnormalities, motor nerve conduction velocity in the fibres supplying the small muscles of the hind paw was reduced to approximately $80 \%$ of the control value.

Histologically, degeneration of axis cylinders and myelin sheaths was found in peripheral nerves, affecting predominantly the distal parts of the longest fibres.

When paralysed animals were no longer given acrylamide, they recovered clinically, conduction velocity returned to the normal range, and there was histological evidence of regeneration of nerve fibres.

Acrylamide $\mathrm{CH}_{2}=\mathrm{CHCONH}_{2}$ has a number of commercial uses, one of which involves pumping solutions containing acrylamide into soil during mining and tunnelling operations in order to render the soil waterproof. It seemed possible that under these conditions men might be seriously exposed to the substance. Tests for its toxicity to experimental animals were therefore undertaken. At the time acrylamide was first introduced, several workers in the pilot plant developed numbness and tingling of the fingers followed by weakness and unsteadiness of the legs. They all recovered within a week when no longer exposed to acrylamide (Golz, personal communication).

When the present work was started, the only available information about the toxicity to animals of acrylamide was one publication by Kuperman (1958) and some unpublished data supplied by the American Cyanamid Company. Kuperman (1958) showed that acrylamide had a strikingly cumulative effect in cats and that chronic poisoning produced reversible ataxia and tremor. He was, however, unable to demonstrate any pathological change in the nervous system. More recently, McCollister, Oyen, and Rowe (1964) have described the clinical effects of acrylamide on rats, guinea-pigs, rabbits, cats, and monkeys. Neurological abnormalities developed in all these species, but again no pathological changes were demonstrated.

The present paper gives more information on the

Received for publication November 19, 1965. toxicity of acrylamide to rats. The clinical syndrome produced by subacute and chronic poisoning, namely ataxia, weakness, and muscle wasting, was suggestive of peripheral neuropathy. We have therefore carried out an electrophysiological and histological study of the peripheral nerves.

\section{Materials and Methods}

A crystalline preparation of acrylamide was used in aqueous solution. Male and female albino rats (Porton strain) of different ages were fed on diet 4IB (Bruce and Parkes, 1956) either as cubes or as powder when acrylamide was added to it as a finely ground powder and mixed for $20 \mathrm{~min}$. in a Hobart food mixer. Single oral doses were given by stomach tube.

Electrophysiological Methods Motor nerve conduction velocity was measured in the fibres supplying the small muscles on the plantar surface of the hind paw, using a technique similar to that originally described for the guinea-pig by Kaeser and Lambert (1962). Details of the method were similar to those described by Fullerton (1966) for the guinea-pig. Basal anaesthesia was produced with intraperitoneal pentobarbitone (Nembutal, Abbot) (30 mg./kg.) and deepened with a halothane and oxygen mixture administered through a facial mask. Body temperature was maintained by heat from a lamp, the intramuscular temperature in the thigh varying between $36^{\circ} \mathrm{C}$. and $39^{\circ} \mathrm{C}$.

The arrangement of stimulating and recording electrodes is shown in Figure ra. Stimulating cathodes consisted of a steel needle inserted through the skin to lie beside the sciatic nerve in the thigh and a spring clip over the course of the posterior tibial nerve at the medial 
a

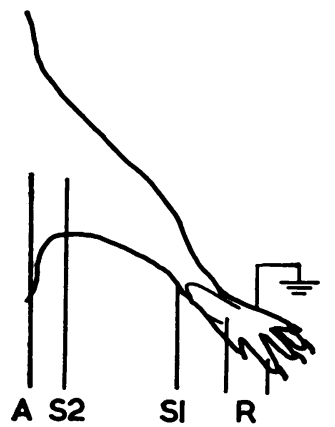

A 52 b

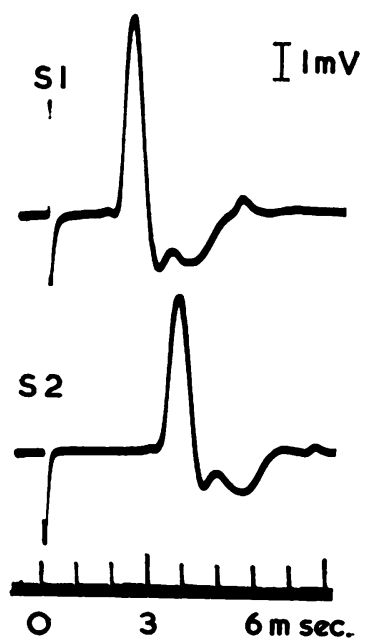

Fig. I. (a) Diagram of electrode arrangement. A, anode; Sr and S2, cathodes; $R$, recording electrodes. (b) Muscle action potentials following nerve stimulation in rat AI6, on 200 p.p.m. acrylamide for five months. Conduction velocity $36 \mathrm{~m} . / \mathrm{sec}$.

side of the ankle. The anode was a clip attached to the skin on the back. The stimulus was a condenser discharge with time constant of 20 or $50 \mu \mathrm{sec}$. delivered through a $I: I$ isolating transformer. Output impedance was less than one kilohm. Stimulus voltage was continuously variable up to 300 volts and was adjusted to be supramaximal for motor fibres.

There are eight interosseous and six lumbrical muscles on the plantar surface of the rat hind-paw, with other small muscles on the lateral and medial borders of the foot (Greene, 1935). Muscle action potentials were recorded through an active electrode, whose tip just penetrated the skin over the middle of the bellies of the interosseous and lumbrical muscles, and a relatively remote electrode on the base of the lateral digit.

Muscle action potentials were amplified and displayed on one beam of a cathode ray oscilloscope, the second beam carrying a time scale. Stimuli were applied at one-second intervals. The distance between stimulating cathodes was measured on the skin and used for the calculation of conduction velocity.

Histological Methods Two or three centimetre lengths of peripheral nerve were removed at necropsy, gently stretched on card frames, and fixed in either $10 \%$ formol saline or Flemming's solution.

Formol-fixed nerves were examined in two ways. Some nerves were dehydrated, embedded in paraffin, and cut in longitudinal sections at $5 \mu$. They were stained by the Holmes silver method to demonstrate axons, and luxol fast blue-cresyl fast violet to demonstrate myelin sheaths (Margolis and Pickett, 1956). Other nerves were stained in $1 \%$ osmium tetroxide and then teased apart in glycerine under a dissecting microscope

(Thomas, 1955). Single fibres were isolated at a magnification of $\times$ 100. Approximately 100 fibres were examined from each nerve, some of these being preserved by mounting them in Canada balsam for examination at higher magnification.

Nerves that were fixed in Flemming's solution were dehydrated and embedded in paraffin, and transverse sections were cut at $5 \mu$. The sections were stained by the modified Weigert method to demonstrate myelin sheaths (Gutmann and Sanders, I943).

For histological examination of the central nervous system rats were perfused with $10 \%$ formol saline. The spinal cord and brain were then removed and fixed in ro $\%$ formol saline for at least one week. Sections were stained with haematoxylin and eosin, cresyl violet, gallocyanin, and by the Marchi method. Bladder tissue was fixed in $10 \%$ formol saline. Longitudinal frozen sections were cut at $80 \mu$ and stained with sudan black B by the method of Cavanagh, Passingham, and Vogt (1964). Other tissues were fixed in Helly's fluid and stained with haematoxylin and eosin.

\section{Results}

Acute Toxicity The single oral dose $\mathrm{LD}_{50}$ for female rats approximately eight weeks old was determined by the method of Weil (1952) and found to be 203 (I66-249) mg. $/ \mathrm{kg}$. After a single dose around the $\mathrm{LD}_{50}$ the rats developed a fine tremor lasting about 48 hours and either recovered completely or died within two to three days showing generalized weakness; in such cases there were no macroscopic lesions at necropsy.

Repeated Doses Although a single oral dose of $100 \mathrm{mg} . / \mathrm{kg}$. produced little more than a fine tremor, if the same dose was repeated 24 hours later most rats died within the next three days showing gross generalized weakness. When the daily dose was reduced to $50 \mathrm{mg} . / \mathrm{kg}$. and given as a series of $\mathrm{I2}$ doses over a period of 15 days, the rats developed severe weakness and died within the next few days. At necropsy many showed gross distension of the bladder, which contained up to $16 \mathrm{ml}$. urine. Male and female rats were equally affected.

In further experiments ro-week-old rats were given $25 \mathrm{mg}$. $/ \mathrm{kg}$. on five days each week. The first perceptible signs of leg weakness developed after 20 doses ( $500 \mathrm{mg}$. $/ \mathrm{kg}$. $/ \mathrm{rat}$ ). This was only evident when animals climbed an inclined board. Severe weakness was present at the time of the twenty-eighth dose, when treatment was stopped. Despite the marked weakness of their legs, these rats were in good general condition and kept themselves well groomed. Within four weeks of acrylamide being stopped, the rats had almost completely recovered.

Daily doses of $10 \mathrm{mg}$. $/ \mathrm{kg}$. had no effect on three female rats aged ro weeks given 55 doses (550 
mg. $/ \mathrm{kg}$.), nor on another three rats aged ro weeks given 116 doses ( $1,160 \mathrm{mg}$. $/ \mathrm{kg}$.).

Another schedule of repeated dosing was tried in which rats were given a single dose of $100 \mathrm{mg} . / \mathrm{kg}$. repeated at different intervals. As stated above, a single dose had only a mild transient effect on the rats, but when it was given on two successive days most rats died.

Groups of six female rats, 3 to 4 months old, were given doses of $100 \mathrm{mg}$. $/ \mathrm{kg}$. acrylamide by mouth at the following intervals:-

Twice Weekly After six doses all the rats had developed marked weakness; four of the six animals were killed and shown to have grossly distended bladders. Treatment was stopped and the others recovered within three weeks.

Once Weekly Two of the six rats died from other causes early in the experiment. After six doses the remaining four showed slight weakness, which became severe after eight doses. Treatment was stopped and the animals had partly recovered when killed three weeks later.

Once in Io Days Six rats showed a varying degree of weakness after seven doses, but treatment was continued in five rats until 24 doses had been given, by which time they showed severe weakness. After the acrylamide had been stopped the rats improved only very slowly, and five months later they still had detectable leg weakness.

Once in I4 Days Slight weakness was first detected in the six rats after the fifteenth dose. One died after the seventeenth dose apparently from lung disease and one was killed after the eighteenth dose as it was very weak. The other four received 28 doses by which time they had progressively developed marked weakness of the legs. When the acrylamide was stopped they showed a slow and incomplete recovery over the next six months after which they were killed.

The long delay before the rats developed signs of severe leg weakness after doses of $100 \mathrm{mg}$. $/ \mathrm{kg}$. given at long intervals could reflect an unusually delayed cumulative effect or it could have arisen because of an increased susceptibility to acrylamide of the older rat. This possibility was investigated by giving rats of different ages two successive daily doses of $100 \mathrm{mg}$. $/ \mathrm{kg}$. The mortality increased from one out of six rats aged $4 \frac{1}{2}$ weeks to five out of six rats aged 13 weeks.

In further experiments, groups of six rats aged $5,8,26$, and 52 weeks were given $100 \mathrm{mg} . / \mathrm{kg}$. acrylamide at weekly intervals. After four doses the youngest animals were only mildly affected whereas those aged 26 weeks were severely affected. The rats aged 52 weeks were severely affected after only three doses. This experiment indicates that older rats are more sensitive to acrylamide given in large doses.

Continuous Feeding Groups of male rats aged 6 to 8 weeks were given diets containing 0,100 , 200,300 , and 400 p.p.m. acrylamide. Some differences in the time taken for the disabilities to develop were observed in different experiments in which the rats were receiving the same concentration in the diet. This appeared to be due partly at least to differences in the quantity of food eaten by the rats, although the reason for this difference was not clear. The daily intake of acrylamide on the various diets was about 6 to $9 \mathrm{mg}$. $/ \mathrm{kg}$. on Ioo p.p.m., Io to I $4 \mathrm{mg}$. $/ \mathrm{kg}$. on 200 p.p.m., I5 to $18 \mathrm{mg}$. $/ \mathrm{kg}$. on 300 p.p.m., and 20 to $30 \mathrm{mg}$. $/ \mathrm{kg}$. on 400 p.p.m. In experiments in which electrophysiological and histological studies were carried out the following observations were made.

Rats on 400 p.p.m. developed slight abnormalities after three weeks, which became severe by eight weeks. Rats on 300 p.p.m. developed slight abnormalities after four weeks, but these became severe only after ro weeks' dosing. On 200 p.p.m. rats showed slight leg weakness after 12 weeks; by 16 weeks this had become marked. They remained in this condition if kept on the same diet for up to another 30 weeks. On IOO p.p.m. the rats only began to show slight leg weakness after 40 weeks on the diet, and this did not increase in the remaining eight weeks of the experiment.

Neurological Features In subacute poisoning produced within one or two weeks the animals showed generalized weakness and were in poor condition. The most striking feature at necropsy was the distended bladder.

When dosing was adjusted to give a milder and more slowly developing lesion, the first sign was a tendency for the animal to keep its hind limbs splayed out when dropped from a few inches onto a flat surface, and the limbs might be held in an unusual position. If acrylamide was continued the rats became increasingly ataxic with difficulty in controlling the limbs. The hind legs dragged along the ground, often with the plantar surface of the hind paw upwards. The abnormal posture of a severely affected animal is shown in Figure 2. Even in the most severely affected animals, however, paralysis was not complete, and there was always some movement in response to pinching the foot. Apart from ataxia and weakness of the limbs the rats were alert and in good general condition. 


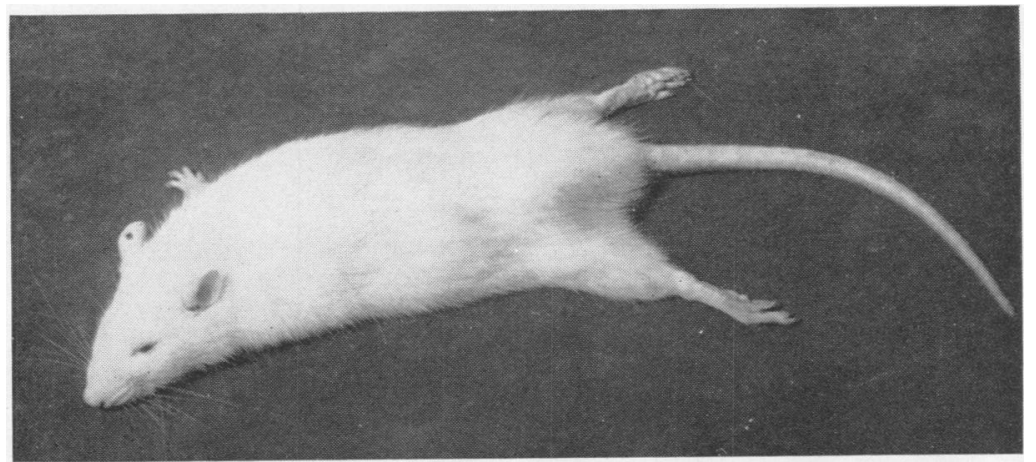

FIG. 2. Weakness and abnormal posture of limbs in rat A7, which had been on a diet containing 400 p.p.m. acrylamide for two months.

When acrylamide was withdrawn the recovery in young animals which had had weakness for only a few weeks was rapid and complete. In the older rats, in which weakness had been present for months, recovery was slow and there was a mild residual ataxia some months later.

Electrophysiological Findings Nerve conduction velocity was measured in the motor fibres supplying the small muscles of the hind paw in ro healthy adult rats, in I I rats at times when they had severe neurological abnormalities of the hind limbs, and in four animals after clinical recovery. Illustrative records of the muscle action potentials recorded from a severely affected animal (AI6) are shown in Figure $\mathrm{Ib}$.

Maximal velocity for the three groups of animals is shown in Figure 3. The mean conduction velocity for the Io control animals was $56 \mathrm{~m}$./sec. (S.D. $5 \cdot 8$ ) and for the poisoned animals it was $44 \mathrm{~m} . / \mathrm{sec}$. (S.D. $2 \cdot 2$ ). The lowest velocity recorded from an affected animal was $37 \mathrm{~m}$./ $/ \mathrm{sec}$. The difference in conduction velocities between the healthy and severely affected rats was highly significant (t test, $P<0.001$ ). Six animals had received 200 p.p.m. acrylamide in their diet for six months, and five had received 400 p.p.m. for two to three months. There was no difference in velocities between the groups of animals on different dose regimes when examined at a comparable stage of paralysis.

In some animals the conduction velocity was measured at frequent intervals during poisoning. The results for one adult rat (A2I) that received 400 p.p.m. acrylamide in its diet throughout the period of observation is shown in Figure 4. It can be seen that there was no change in conduction velocity during the first six weeks of dosing, by which time the animal had developed mild ataxia. However, by the time the animal was severely affected after nine weeks, maximal conduction velocity had fallen from $57 \mathrm{~m}$. $/ \mathrm{sec}$. to $42 \mathrm{~m}$./ $/ \mathrm{sec}$., a fall of $26 \%$.

Conduction velocity was measured in four animals after recovery from severe paralysis. These animals had returned to a normal diet five to nine months previously. Mean conduction velocity was

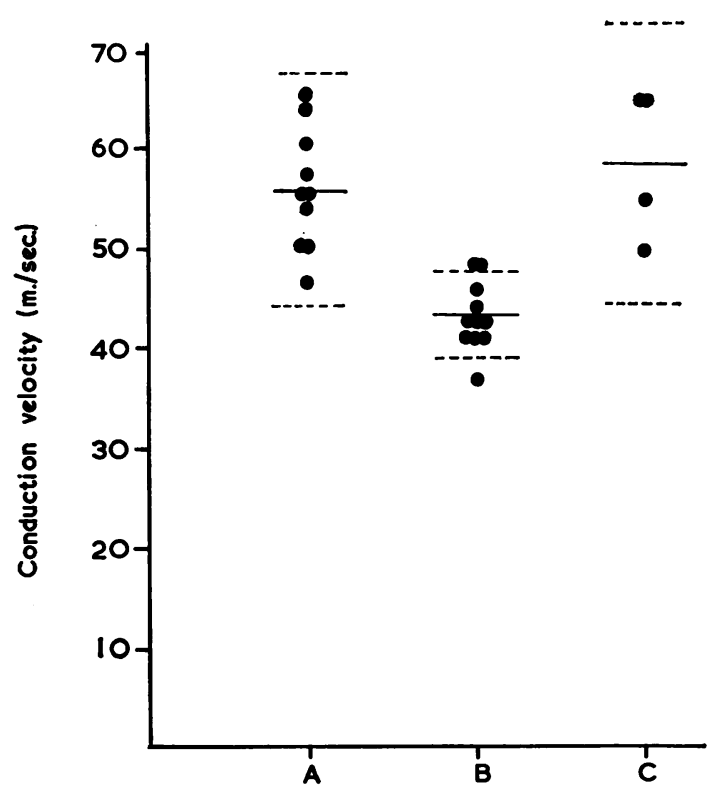

FIG. 3. Maximal nerve conduction velocity in motor fibres to the hind paw in (A) healthy adult rats; (B) severely affected rats which had been on a diet containing 200 p.p.m. acrylamide for six months or 400 p.p.m. for two to three months; (C) rats which had been severely affected and returned to a normal diet five to nine months previously. Solid lines indicate mean conduction velocities; dotted lines are two standard deviations from the mean. 


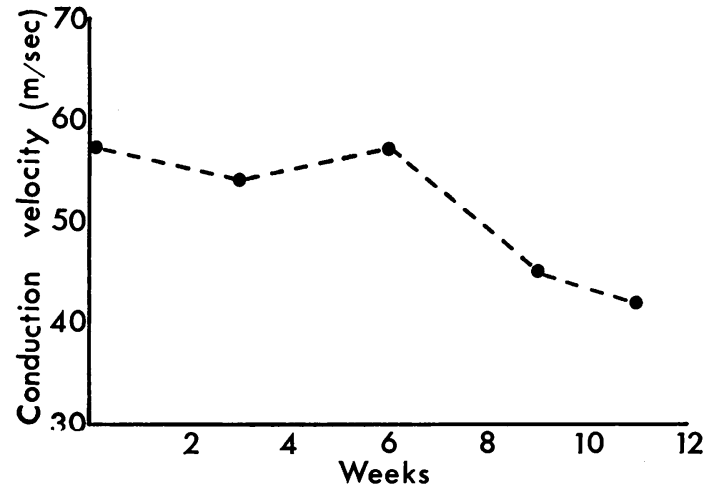

FIG. 4. Conduction velocity in rat A2I while on a diet containing 400 p.p.m. acrylamide.

$59 \mathrm{~m} . / \mathrm{sec}$. (S.D. 7.0) (Fig. 3 C). This value is not significantly different from the value for healthy adult rats ( $t$ test, $P>0.4$ ), but it is significantly faster than the value for the severely affected group (t test, $\mathrm{P}<0.00 \mathrm{I})$.

Pathological Findings Apart from gross bladder distension in animals with subacute poisoning, the only macroscopic lesion at necropsy was wasting of the muscles of the hind limbs seen in the chronically poisoned animals. Histological examination of the kidney, spleen, pancreas, suprarenal, and lungs showed no abnormalities in animals with acute, subacute, or chronic poisoning. In animals that died from acute poisoning, fine fatty infiltration of the liver was present; however, there were no abnormalities of the livers of chronically poisoned animals.

No histological abnormality was demonstrated in the brain or spinal cord of acutely or chronically poisoned rats.

Bladders were examined from animals that had developed retention of urine and were compared with those from control rats. No abnormality of the myelinated fibres was demonstrated by Sudan black B staining.

Peripheral nerves from 17 animals that had been poisoned with 200,300 , or 400 p.p.m. acrylamide in their diets for periods of one to six months were examined histologically. All the animals had severe clinical abnormalities. In addition, three rats were returned to a normal diet after receiving 300 p.p.m. acrylamide for four months and were killed six months later.

There was extensive degeneration both of axis cylinders and of myelin sheaths in the peripheral nerves of all the clinically affected animals. The appearances were similar to those seen during Wallerian degeneration. This process will be referred to as axonal degeneration. Only two short lengths of segmental demyelination were seen during examination of many hundreds of nerve fibres from affected animals. Although such changes were not seen in nerves from three control rats, the importance of these findings is very doubtful. Lubinska (1958) has described similar appearances in occasional nerve fibres from healthy cats.

Figure 5 shows three single fibres at different stages of axonal degeneration. The myelin sheath of the fibre shown in Fig. 5a had broken into irregular lengths and there was some retraction of myelin at intervals along the fibre. In the fibre seen in Fig. $5 \mathrm{~b}$, the process was more advanced and there were longer gaps in the myelin. The fibre illustrated in Fig. $5 \mathrm{c}$ was at an even later stage of degeneration, the myelin having formed ovoids at irregular intervals.

Changes in axons as well as myelin sheaths were seen in longitudinal sections of peripheral nerve stained by the Holmes silver method with luxol fast blue-cresyl fast violet. Figure 6 shows a degenerating fibre from an animal that had been fed 400 p.p.m.

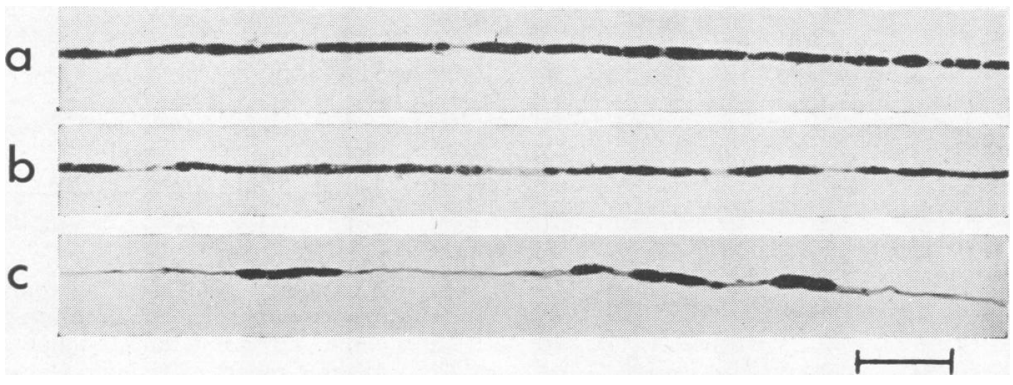

FIG. 5. Teased single fibres stained with $I \%$ osmium tetroxide showing stages of fragmentation of myelin during axonal degeneration: (a) and (b) from rat AII ; (c) from rat A12. Both rats on diet containing 400 p.p.m. acrylamide for two months. Calibration $100 \mu$. 


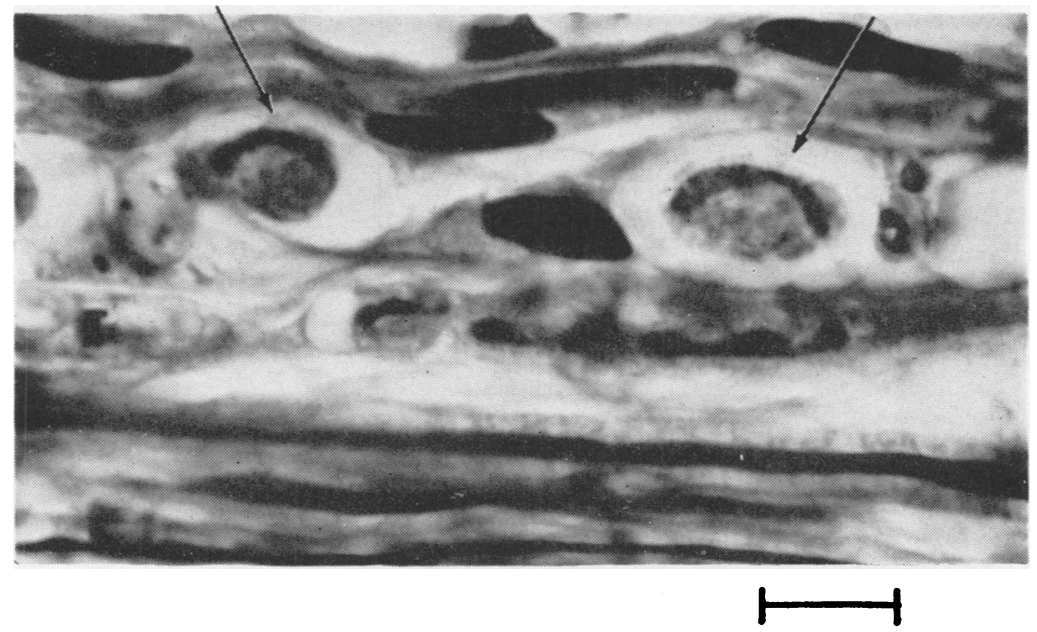

FIG. 6. Longitudinal section of posterior tibial nerve from rat AII, which had been on 400 p.p.m. acrylamide for two months. Staining by the Holmes silver method with luxol fast blue-cresyl fast violet. A normal fibre can be seen at the bottom of the figure. Two myelin ovoids from a degenerating fibre are indicated by arrows. Calibration ro $\mu$.

acrylamide in its diet for two months. Degeneration of the fibre had reached the stage of formation of ovoids. Darkly staining segments of broken-up axon can be identified within the paler staining myelin ovoid.

Distribution of Lesion The proportion of abnormal myelinated fibres in the nerves was assessed from transverse sections fixed in Flemming's solution and stained to show myelin. Examination of sections taken at different levels of the nerve trunks has shown that the peripheral parts of the nerves were always more severely affected than the proximal segments. This is illustrated in Figs. 7 and 8 from a rat that had been fed 200 p.p.m. acrylamide for five months. Sections of ventral and dorsal roots, sciatic nerve in the thigh, posterior tibial nerve at the ankle, and sural nerve in the calf are shown in Figure 7. It can be seen that the density of fibres in the sciatic nerve was less than in the ventral or dorsal roots. The density was even lower in the posterior tibial and sural nerves. Parts of the sections from Fig. 7 are shown at higher magnification in Fig. 8 compared with similar sections from a healthy rat. The sections have been mounted in pairs with the control nerve on the right and the nerve from the poisoned animal on the left in each case. The ventral root from the poisoned animal (A) was comparable to that from the control (B). Occasional degenerating fibres were present in the dorsal root (C), but there was no apparent decrease in the density of fibres. However, there was an obvious reduction in the density of fibres in the sciatic nerve from the affected animal (E) compared with that from the control $(F)$. The reduction in density was even more marked when the posterior tibial ( $G$ and $H$ ) and sural ( $I$ and $J$ ) nerves were compared. Since the sural nerve contains only sensory fibres, there is thus clear evidence of sensory nerve involvement.

There was no apparent reduction in density of myelinated nerve fibres in transverse sections of dorsal and ventral roots from seven animals. When roots from three animals were examined by teasing of single fibres, occasional fibres were seen to be undergoing axonal degeneration in both dorsal and ventral roots. The changes were, however, scanty compared with the severe degree of abnormality in the posterior tibial and sciatic nerves from these animals.

Median or ulnar nerves from the forelimb were examined from four animals. All the nerves contained fibres undergoing axonal degeneration. In one animal examination of transverse sections showed that the degree of abnormality was similar in the median nerve in the forelimb and the sciatic nerve in the thigh.

Fibre Diameter The diameter of all the myelinated fibres in the posterior tibial nerve was measured in two control animals and in four animals that had received acrylamide. Photographs of sections of nerves fixed in Flemming's solution and stained by the modified Weigert method were 

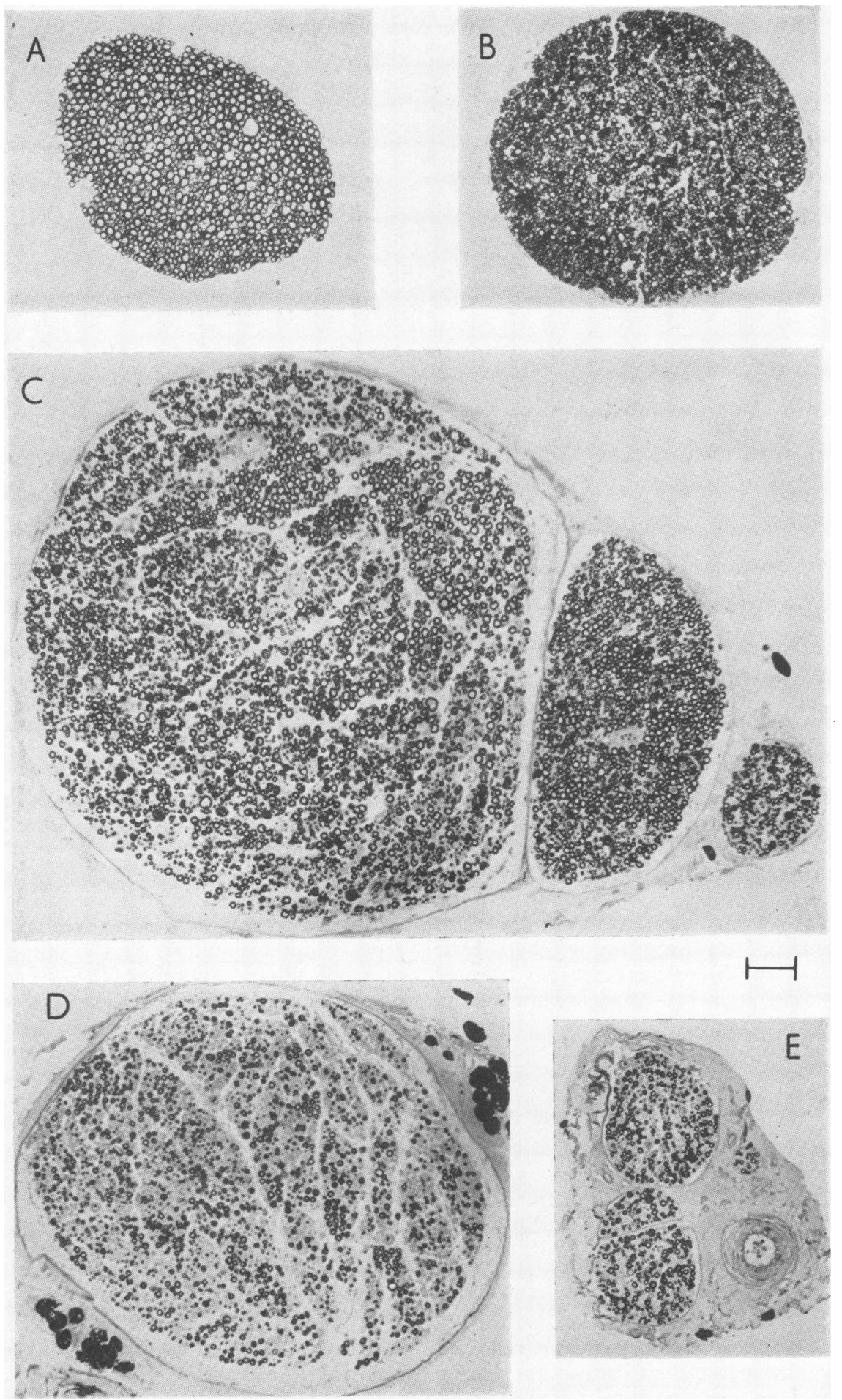

FIG. 7. Transverse sections of nerves fixed in Flemming's solution and stained by the modified Weigert method. Rat A13, on 200 p.p.m. acrylamide for six months. (A) Lumbar ventral root; (B) lumbar dorsal root; (C) sciatic nerve in thigh; (D) posterior tibial nerve above ankle; (E) sural nerve in mid calf. Calibration $100 \mu$. 

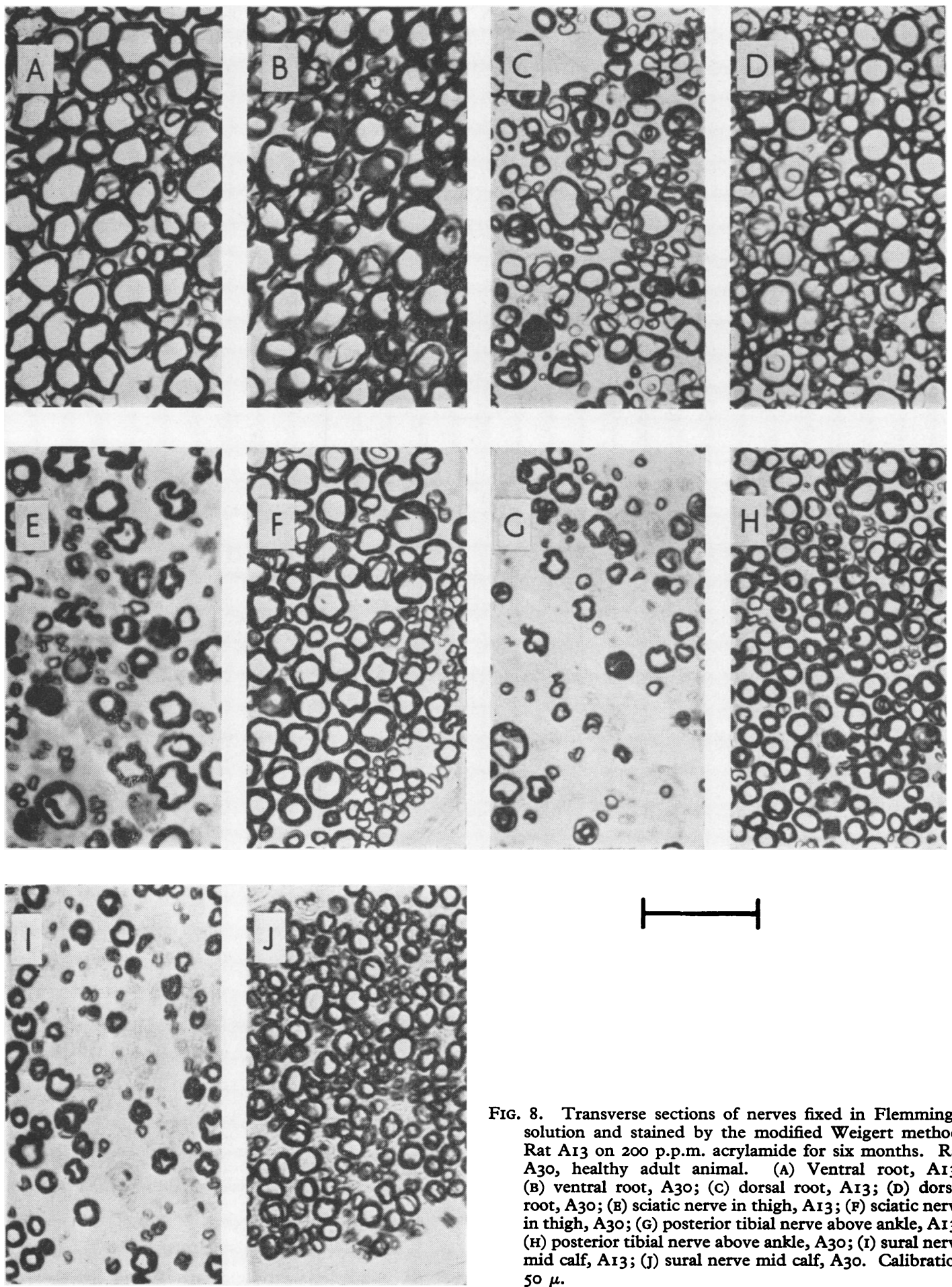

FIG. 8. Transverse sections of nerves fixed in Flemming's solution and stained by the modified Weigert method. Rat Ar3 on 200 p.p.m. acrylamide for six months. Rat A30, healthy adult animal. (A) Ventral root, Ar3; (B) ventral root, $\mathrm{A}_{30}$; (C) dorsal root, $\mathrm{Ar}_{3}$; (D) dorsal root, A30; (B) sciatic nerve in thigh, Ar 3 ; (F) sciatic nerve in thigh, A30; (G) posterior tibial nerve above ankle, AI3; (H) posterior tibial nerve above ankle, $A_{30}$; (I) sural nerve mid calf, Ar3; (J) sural nerve mid calf, A30. Calibration $50 \mu$. 


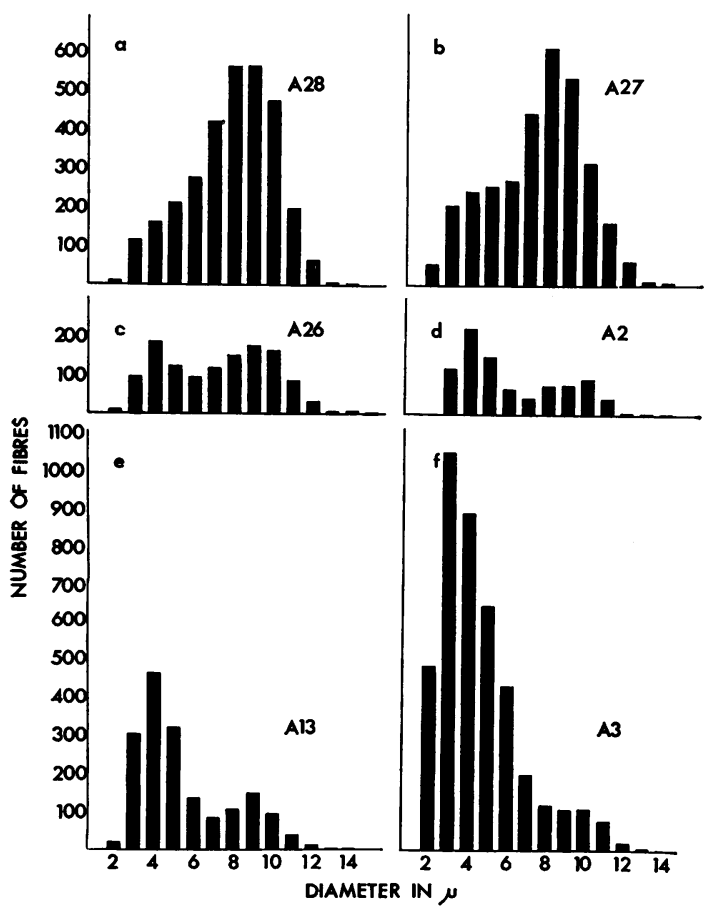

FIG. 9. Histograms showing distribution of diameters of all myelinated fibres in posterior tibial nerves of animals on the following diets: (a) and (b) healthy adult rats; (c) 400 p.p.m. acrylamide for two months; (d) 300 p.p.m. acrylamide for four months; (e) 200 p.p.m. acrylamide for six months; (f) 300 p.p.m. acrylamide for four months, followed by normal diet for six months.

used for measurement, as described by Thomas and Fullerton (1963). Photographs were taken at $\times 250$ magnification and enlarged to a final magnification of $\times 1,000$.

Histograms of fibre diameter are shown in Figure 9. In the control animals it can be seen that there was a unimodal distribution of diameter, with a peak at 8 to $9 \mu$. The rat that had received 400 p.p.m. acrylamide in its diet for two months and the one that had received 300 p.p.m. for four months had severe neurological abnormalities. The 8 to $9 \mu$ diameter peak has disappeared from both histograms, suggesting that fibres of this diameter were the most severely affected.

Regeneration Many regenerating fibres were found in nerves from animals that were returned to a normal diet after developing severe paralysis. Figure of shows the histogram of fibre diameter of the posterior tibial nerve of rat $\mathrm{A}_{3}$, which had received a diet containing 300 p.p.m. acrylamide for four months and had then been returned to a normal diet for six months. No neurological abnormalities were present when it was killed. It can be seen that there were many more fibres with a diameter of 3 to $4 \mu$ than in the control animals, suggesting that regeneration might have been occurring.

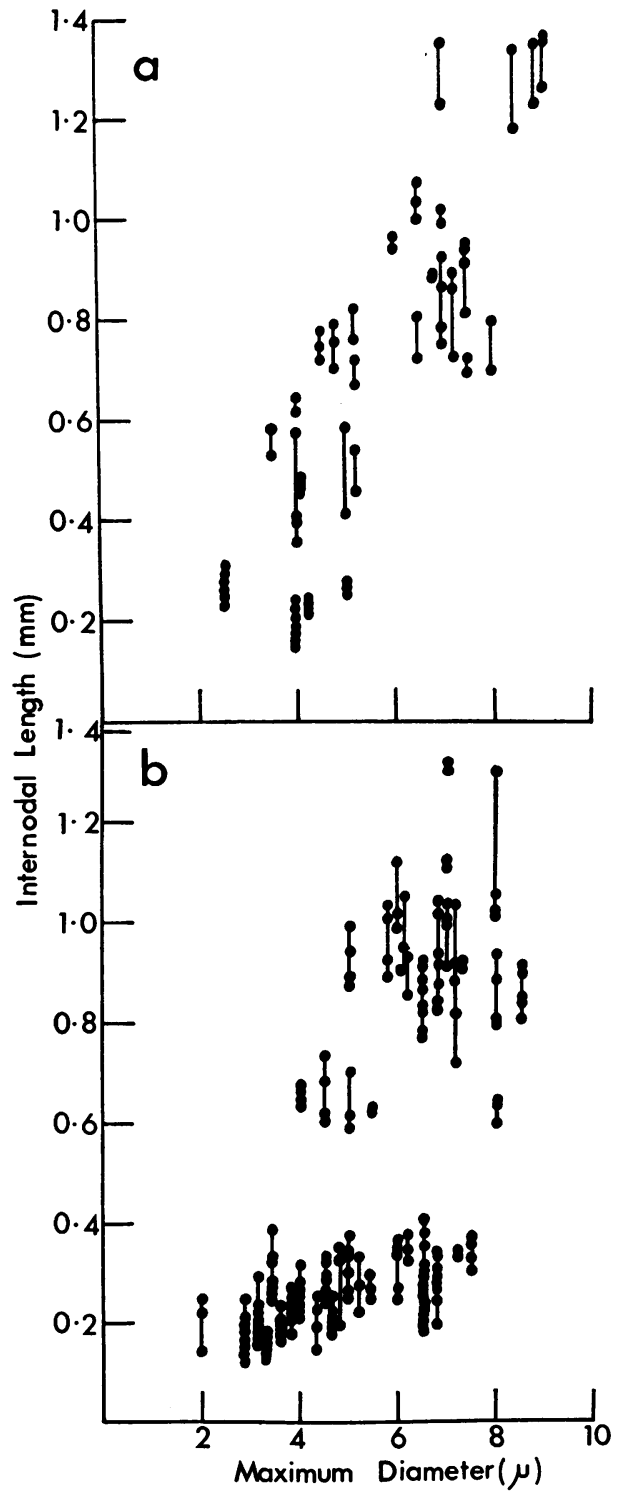

FIG. 1o. Internodal length and fibre diameter in posterior tibial nerve of (a) normal rats, (b) rats $\mathrm{A}_{3}$ and $\mathrm{A}_{4}$ on 300 p.p.m. acrylamide for four months, followed by normal diet for five to six months. Values for different internodal lengths of the same fibre are joined by a line. These are plotted against the diameter of the largest internodal segment of the fibre concerned. 
The presence of regenerating fibres in nerves from this animal and from another similarly treated (A4) was confirmed by measuring the fibre diameter and internodal length for teased single fibres. Vizoso and Young (1948) have shown that in normal rabbit nerves there is a linear relationship between the internodal length and fibre diameter, but in regenerated fibres this relationship is lost and fibres of all diameters have short internodal lengths. A similar relationship between the fibre diameter and internodal length for the posterior tibial nerve from two healthy rats was demonstrated and is illustrated in Figure roa. Values for different internodal segments have been joined by a line, and the diameter given for each fibre is that of the largest internodal segment in the fibre concerned. This method of presenting the results is similar to that described by Fullerton, Gilliatt, Lascelles, and Morgan-Hughes (1965).

Figure Iob shows similar measurements for single fibres from rats $A_{3}$ and $A_{4}$. It can be seen that a proportion of fibres with diameters from 5 to $8 \mu$ have shorter internodal lengths than any normal fibres of the same diameter. These therefore may be presumed to be fibres that have regenerated after axonal degeneration.
Examples of a normal and regenerated fibre from rat $\mathrm{A}_{4}$ are shown in Fig. II; Figs. $\mathrm{I}$ Ia to $\mathrm{d}$ are continuous lengths of a normal fibre mounted one below the other. The diameter of the largest internodal segment of this fibre was $7 \mu$, and the internodal length of five segments ranged from $0.65 \mathrm{~mm}$. to $0.87 \mathrm{~mm}$. Figures $\mathrm{IIe}$ and $\mathrm{f}$ are consecutive lengths of a regenerated fibre. The diameter of the largest internodal segment was $6.5 \mu$. The internodal length of four segments was only 0.28 to $0.32 \mathrm{~mm}$. From Fig. IOa it can be seen that normal fibres with a diameter of $6.5 \mu$ would be expected to have internodal lengths of 0.7 to $\mathrm{I} \cdot \mathrm{I} \mathrm{mm}$.

Some evidence of regeneration was found in rats on low doses of acrylamide, even when this was continued until they were killed. For example, rat Ar3 had been on a diet containing 200 p.p.m. acrylamide for six months, and its hind limbs were severely affected. The histogram of fibre diameter for the posterior tibial nerve is shown in Figure 9e. The reduction in the number of fibres in the 8 to $9 \mu$ range is comparable to that in the nerves from animals that had received 300 p.p.m. and 400 p.p.m. acrylamide (Figs. 9c and d). However, there appeared to be an increase in the number of small diameter fibres compared with the control animals,

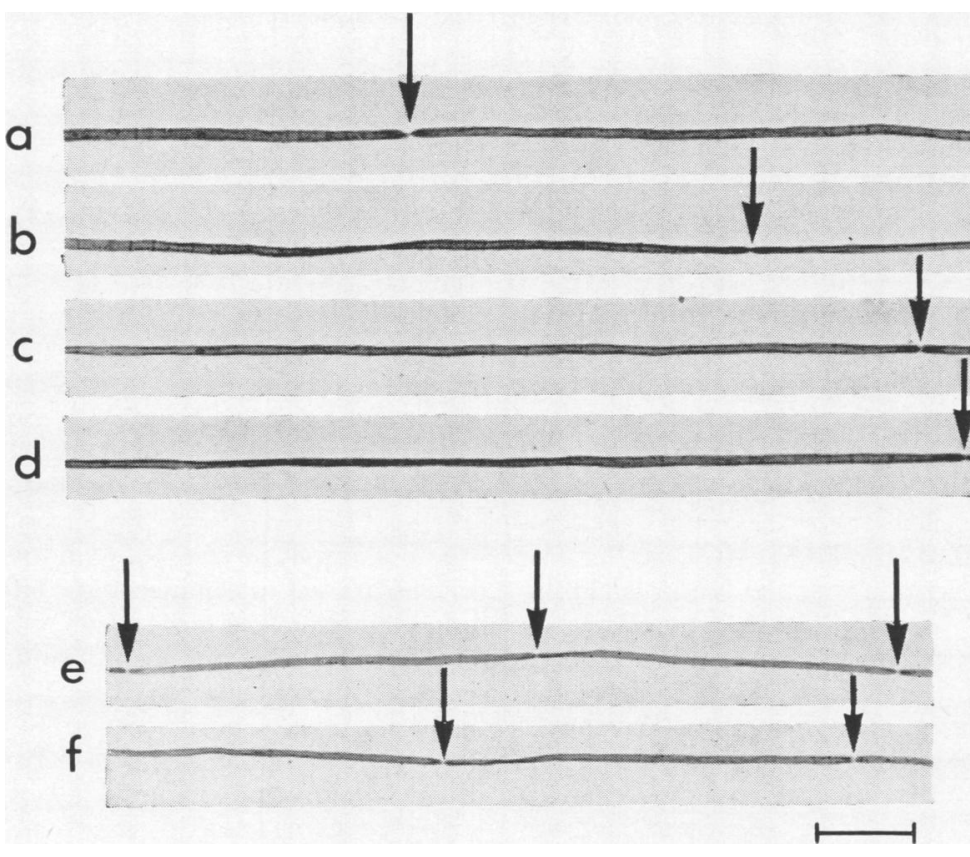

Frg. II. Two single fibres, stained with I\% osmium tetroxide from rat A4, on 300 p.p.m. acrylamide for four months followed by a normal diet for six months. Arrows indicate nodes of Ranvier. (a to d) Continuous lengths of a normal fibre; ( $e$ and $f$ ) continuous lengths of a regenerated fibre. Calibration $100 \mu$. 
suggesting that regeneration may have occurred although poisoning had been continued without interruption.

Further evidence of regeneration was therefore sought in the nerves from rat Ar3. In fibres with diameters of less than $5 \mu$, internodal lengths are similar for normal and regenerating fibres (Fig. IO). It was therefore not possible to confirm by this method of measurement that the small diameter fibres in nerves from rat $\mathrm{Al}_{3}$ were regenerating. However, when single fibres were examined, a few of the small diameter fibres were seen to have myelin ovoids attached to them. It seems probable that these fibres were regenerating and that the remnants of degenerating myelin had persisted since degeneration of the original fibre within the same endoneurial sheath.

\section{Discussion}

McCollister et al. (1964) have given a full account of the toxicity of acrylamide and shown that most species react in a similar way. Unpublished observations in this laboratory have shown that mice are susceptible, and we have confirmed the findings in cats. McCollister and his colleagues (1964) drew attention to the prominence of the neurotoxic effects in all species but had no evidence about the nature of the lesion. Kuperman (1958) made a detailed physiological study of poisoned cats and concluded that the most likely site of action of acrylamide given in doses that produced acute poisoning was on the mesencephalic tegmentum of the brain stem. He did not, however, demonstrate any pathological changes in his animals.

It has now been shown that the neurological abnormalities which develop during chronic poisoning are due to peripheral neuropathy. Both motor and sensory nerves are affected. Reduction of conduction velocity in the motor fibres supplying the hind-paw indicates that motor fibres are involved, and pathological abnormalities were demonstrated in the purely sensory sural nerve. Axonal degeneration occurs and is most marked at the distal end of the longest fibres. In this way the lesion is similar to the 'dying back' process discussed by Cavanagh (1964a), which has previously been demonstrated in animals with thiamin deficiency (Swank, 1940) and tri-ortho-cresyl phosphate poisoning (Cavanagh, 1964b). In all instances, histological examination of the nerve cells themselves has only shown changes of chromatolysis. It has been suggested by both Swank and Cavanagh that the basic biochemical defect might nevertheless be in the cell body. The supply of some essential metabolite might be defective at the distal ends of the longest fibre due to inadequate synthesis in the cell body. Cavanagh (1964a) has pointed out that this type of pathological change is common in human disease also (e.g., motor neurone disease and Friedreich's ataxia).

Axonal degeneration as described here is a distinct process which differs from segmental demyelination, the other type of pathological change that may occur in peripheral nerves. In the latter process there is patchy breakdown of myelin affecting individual internodal segments, the axis cylinders remaining in continuity. Segmental demyelination has been shown to occur in lead neuropathy (Gombault, I880), experimental allergic neuritis (Waksman and Adams, 1956), and diphtheritic neuropathy (Waksman, Adams, and Mansmann, 1957).

In rats with severe abnormalities due to acrylamide, the conduction velocity of the most rapidly conducting motor nerve fibres was reduced compared with that of the control animals. The reduction of maximal velocity was, however, slight, the mean being only $20 \%$ lower in the group of poisoned animals than in the control animals. The range of conduction velocities in the motor fibres supplying the small muscles of the rat hind paw is not known, but there is likely to be a difference of at least $20 \%$ between the fastest and slowest conducting fibres in the nerve. The reduction in conduction velocity in the poisoned animals could therefore be explained by failure of conduction in the fastest fibres, unmasking normal fibres that conduct at a slower velocity. This finding is in contrast to the much more marked reduction of conduction velocity that has been demonstrated in neuropathies in which pathological changes of segmental demyelination are found. In such cases conduction velocity may fall to 10 to $20 \%$ of the normal figure. For example, McDonald (1962, 1963), Kaeser and Lambert (1962), and Morgan-Hughes (1965) have observed marked slowing of conduction in diphtheritic neuropathy. Cragg and Thomas (1964) and Hall (1964) found similar changes in experimental allergic neuritis, and Fullerton (1966) has also demonstrated slowing of conduction in the peripheral nerves of guinea-pigs in which segmental demyelination was produced by lead poisoning.

There have been few studies of nerve conduction in experimental neuropathies in which axonal degeneration is the sole finding. Kaeser (1962) found little change in maximal conduction velocity in thallium intoxication in guinea-pigs with extensive axonal degeneration in peripheral nerves. During Wallerian degeneration following nerve section, Gutmann and Holubár (1950) found that conduction velocity was $87 \%$ of the control value just before conduction failed completely, and similar 
findings have been reported by Kaeser (1962). The preservation of relatively normal conduction velocity in rats with severe acrylamide neuropathy is in accordance with these observations.

Bladder distension was a common finding in subacutely poisoned animals. In view of the widespread peripheral nerve damage produced by acrylamide, it seems probable that the bladder paralysis also has a neurogenic basis. No abnormality of myelinated fibres in the bladder has been demonstrated, but it is known that the motor innervation of the cat's bladder is by non-myelinated fibres (Kleyntjens and Langworthy, 1937). It seems possible therefore that a more detailed examination of non-myelinated fibres might demonstrate some abnormality of bladder innervation in poisoned rats.

The striking cumulative effect of acrylamide is difficult to explain for a compound of low molecular weight and free solubility in water. It cannot be due to an accumulation of the compound itself in the tissue, although a cumulative metabolic effect might occur. Neurological signs may, however, only become evident when there has been failure of conduction in a relatively high proportion of peripheral nerve fibres. For example, in man it has been suggested that a loss of $30 \%$ to $40 \%$ of the sensory fibres may occur before there is any detectable sensory loss (Gilliatt and Wilson, 1954). If each small dose of acrylamide damages only a few nerve fibres, then a delay in onset of neurological signs might be expected.

A further possible explanation for the apparent cumulative effect is the increased susceptibility of older rats to the toxic action of acrylamide. Such an effect has been demonstrated for both acute and chronic poisoning. Thus, doses which produce no clinical abnormality in young rats produce neurological signs after several months, merely because the animal is then older and more susceptible to the same dose.

In rats given low doses of acrylamide in the diet, it has been shown that regeneration of some of the nerve fibres was probably taking place even while they were still receiving acrylamide. Thus, a further factor contributing to the delayed development of neurological signs might be a change in the balance between the amount of neurone damage being produced and the rate at which injured neurones recover. It is possible that as rats grow older their capacity for repair diminishes.

Nothing is known about the biochemical dis- turbances produced by acrylamide either in the whole animal or in isolated biological systems. The investigations described above now offer an explanation of the disabilities seen in chronically poisoned animals, and investigations into the metabolic disturbances produced by acrylamide are clearly called for.

We should like to thank Dr. Boyd Shaffer, American Cyanamid Co., for some unpublished data on acrylamide; Professor R. W. Gilliatt for his advice and criticism; Dr. $P$. N. Magee for reports on the pathology of the viscera and help with examination of the central nervous system and C. R. Kennedy and P. Forshaw for technical assistance.

\section{REFERENCES}

Bruce, H. M., and Parkes, A. S. (1956). Letter, F. Anim. Tech. Ass., 7, 54.

Cavanagh, J. B. (1964a). Int. Rev. exp. Path., 3, 219. (I964b). F. Path. Bact., 87, 365.

—, Passingham, R. J., and Vogt, J. A. (I964). Ibid., 88, 89.

Cragg, B. G., and Thomas, P. K. (1964). f. Neurol. Neurosurg. Psvchiat., 27, 106.

Fullerton, P. M. (1966). f. Neuropath. exp. Neurol., 25, 214.

-, Gilliatt, R. W., Lascelles, R. G., and Morgan-Hughes, J. A. (1965). F. Physiol. (Lond.), 178, 26 P.

Gilliatt, R. W., and Wilson, T. G. (I954). F. Neurol. Neurosurg. Psychiat., 17, 104.

Golz, H. H. Personal communication.

Gombault, M. (1880). Arch. Neurol. (Paris), I, II.

Greene, E. C. (1935). Trans. Amer. Philos. Soc., n.s. 27.

Gutmann, E., and Holubář, J. (1950). F. Neurol. Neurosurg. Psychiat., 13, 89.

- and Sanders, F. K. (1943). F. Physiol. (Lond.), 101, 489.

Hall, J. I. (1964). Electroenceph. clin. Neurophysiol., 17, 704.

Kaeser, H. E. (1962). Dtsch. Z. Nervenheilk., 183, 268.

- and Lambert, E. H. (1962). Electroenceph. clin. Neurophysiol., Suppl. 22, p. 29.

Kleyntjens, F., and Langworthy, O. R. (1937). F. comp. Neurol., 67, 367.

Kuperman, A. S. (1958). F. Pharmacol. exp. Ther., 123, 180. Lubińska, L. (1958). Nature, 181, 957.

McCollister, D. D., Oyen, F., and Rowe, V. K. (1964). Toxicol. appl. Pharmacol., 6, 172.

McDonald, W. I. (1962). Acta neuropath. (Berl.), 1, 425. - (1963). Brain, 86, 501.

Margolis, G., and Pickett, J. P. (1956). Lab. Invest., 5, 459.

Morgan-Hughes, J. A. (1965). Rev. Patol. nerv. ment., 86, 253.

Swank, R. L. (1940). F. exp. Med., 71, 683.

Thomas, P. K. (1955). Proc. roy. Soc. B., 143, 380.

- and Fullerton, P. M. (1963). F. Neurol. Neurosurg. Psychiat., 26, 520.

Vizoso, A. D., and Young, J. Z. (1948). F. Anat. (Lond.), 82, 1 I0.

Waksman, B. H., and Adams, R. D. (1956). F. Neuropath. exp. Neurol., 15, 293.

, $\frac{1}{59}$ and Mansmann, H. C. (1957). F. exp. Med., 105, 591.

Weil, C. S. (1952). Biometrics, 8, 249. 